\title{
Review Article \\ Endovascular Intervention for Acute Ischemic Stroke in Light of Recent Trials
}

\author{
Kenan Alkhalili, ${ }^{1}$ Nohra Chalouhi, ${ }^{2}$ Stavropoula Tjoumakaris, ${ }^{2}$ David Hasan, ${ }^{3}$ \\ Robert M. Starke, ${ }^{4}$ Mario Zanaty, ${ }^{2}$ Robert H. Rosenwasser, ${ }^{2}$ and Pascal Jabbour ${ }^{2,5}$ \\ ${ }^{1}$ Department of Surgery, University of Arizona, Tucson, AZ 85721, USA \\ ${ }^{2}$ Department of Neurosurgery, Thomas Jefferson University and Jefferson Hospital for Neuroscience, Philadelphia, PA 19107, USA \\ ${ }^{3}$ Department of Neurosurgery, University of Iowa, Iowa City, IA 52242, USA \\ ${ }^{4}$ Department of Neurosurgery, University of Virginia, Charlottesville, VA 22908, USA \\ ${ }^{5}$ Department of Neurological Surgery, Division of Neurovascular Surgery and Endovascular Neurosurgery, \\ Thomas Jefferson University Hospital, 901 Walnut Street, 3rd Floor, Philadelphia, PA 19107, USA
}

Correspondence should be addressed to Pascal Jabbour; pascal.jabbour@jefferson.edu

Received 5 July 2014; Accepted 16 August 2014; Published 3 November 2014

Academic Editor: Stephen J. Monteith

Copyright (C) 2014 Kenan Alkhalili et al. This is an open access article distributed under the Creative Commons Attribution License, which permits unrestricted use, distribution, and reproduction in any medium, provided the original work is properly cited.

Three recently published trials, MR RESCUE, IMS III, and SYNTHESIS Expansion, evaluating the efficacy and safety of endovascular treatment of acute ischemic stroke have generated concerns about the future of endovascular approach. However, the tremendous evolution that imaging and endovascular treatment modalities have undergone over the past several years has raised doubts about the validity of these trials. In this paper, we review the role of endovascular treatment strategies in acute ischemic stroke and discuss the limitations and shortcomings that prevent generalization of the findings of recent trials. We also provide our experience in endovascular treatment of acute ischemic stroke.

\section{Introduction}

About 795000 people experience a new or recurrent stroke in the United States on annual basis [1]. Ischemic strokes represent $85 \%$ of cases and are frequently caused by occlusion of larger arteries [1-3]. The role of endovascular therapy in the treatment of acute ischemic stroke (AIS) must be carefully considered, given the remarkable advancement that imaging and endovascular treatment modalities have undergone over the past several years. Recent publication of the Interventional Management of Stroke (IMS) III [4], Mechanical Retrieval and Recanalization of Stroke Clots Using Embolectomy (MR RESCUE) [5], and SYNTHESIS Expansion [6] trials brought into question the clinical value of endovascular therapy in acute ischemic stroke (AIS). These reports, however, have several significant weaknesses and do not reflect the recent advances in imaging and endovascular technology.
The endovascular treatment of AIS has advanced significantly, with notable transition from intra-arterial (IA) chemical thrombolysis to mechanical thrombectomy [7-10]. First-generation devices, such as the Merci (Stryker; Kalamazoo, MI, USA) and Penumbra (Penumbra Inc., Alameda, CA, USA) devices, were developed to engage or aspirate the clot in different means [11]. Aspiration through the balloon guide catheter is applied during clot retrieval. The secondgeneration devices (e.g., "stentrievers" such as Solitaire (Covidien/ev3, Dublin, Ireland) or Trevo (Stryker, Kalamazoo, MI, USA)) have unique dual functionality, acting as a temporary bypass providing immediate flow restoration through the thrombus and as a clot retriever, trapping thrombus into its cells $[12,13]$.

The effect of stent retrievers on acute stroke treatment is currently being investigated in several ongoing multicenter trials such as the STAR Trial (Solitaire FR Thrombectomy for 
Acute Revascularization), the THRACE trial, and the RIVER II trial.

\section{IMS III}

2.1. Design. IMS III trial is an international, phase 3, randomized, open-label clinical trial with a blinded outcome that tested the approach of intravenous recombinant tissue plasminogen activator (rtPA) followed by protocol-approved endovascular treatment, as compared with standard intravenous rtPA.

The trial hypothesized that combined IV-IA approach ("bridging") for recanalization of acute stroke is superior to standard IV approach.

2.2. Results. Functional independence scores did not differ between the endovascular group and IV rtPA cohorts (modified Rankin scores [mRS] of $0-2: 40.8 \%$ versus $38.7 \%$ ) as did mortality rates $(19.1 \%$ and $21.6 \%, P=0.52)$ at 3 -month follow-up. Rates of symptomatic intracranial hemorrhage (ICH) were similar between both groups refuting safety concerns about endovascular therapy. On the other hand, in a subgroup analysis in patients with confirmed large vessel occlusion (LVO), combined therapy was superior in terms of recanalization and outcomes to IV $\operatorname{rtPA}$ alone $(P=0.01)$.

2.3. Flaws. A number of weaknesses have been observed in this trial [14-16]. To begin with, during the 6-year long inclusion period, imaging and endovascular treatment modalities underwent major advancement, and utilization of new technologies was only partially adopted. Only $47 \%$ of the patients had CT angiogram (CTA) imaging at the time of enrollment, since the National Institute of Health Stroke Scale (NIHSS) score alone is not an accurate enough predictor of LVO. Subsequently, patients without confirmed LVO have been enrolled in the trial. Twenty percent of patients enrolled in the endovascular arm had no LVO on angiography; these patients were still considered in IA arm for trial purposes.

Another limitation of IMS trial is that most patients in the endovascular arm received IA thrombolysis or thrombectomy using Merci retriever. Both approaches are currently considered outdated for endovascular treatment of AIS. The first-generation thrombectomy devices used in the trial are known to be inferior to stent retrievers in terms of recanalization and outcome $[17,18]$. Thus, the results of IMS III did not reflect the huge advances that occurred in endovascular technology over the past few years.

In addition, the significant delay of more than 2 hours between initiation of IV and IA merits attention. This delay may have a deleterious effect on the IA intervention efficacy. Moreover, most of the patients enrolled in IA arm received less than the standard dose of IV rtPA before undergoing angiography, and although all patients in the IV arm received standard doses, we should also mention the selection bias that limited IA treatment to only IV Tpa eligible patients, where in real life the majority of patients eligible for IA thrombectomy did not meet criteria for IV tPA.
2.4. Strengths. This trial is considered to be the largest randomized stroke trial conducted so far. Nine hundred subjects were planned to demonstrate the superiority of combined approach. After the enrolment of 656 participants, the study was halted because interim analysis showed futility (low probability of finding significant difference between both groups). However, when only patients with confirmed LVO were considered, there was a significant benefit for endovascular therapy in terms of recanalization and outcomes. This is probably the single most important conclusion of the IMS III trial. Table 1 summarizes recently published randomized trials of acute stroke therapies.

\section{MR RESCUE}

3.1. Design. MR RESCUE was a phase $2 \mathrm{~b}$, randomized, controlled, open-label (blinded outcome), multicenter trial. The trial hypothesized that patients with favorable neuroimaging "penumbral" pattern were more likely to achieve better outcome from IA treatment of AIS. Penumbral pattern was defined as the presence of substantial salvageable tissue with small infarct core (predicted infarct core $\leq 90 \mathrm{~mL}$ and ratio of predicted infarct tissue within the at-risk region $\leq 70 \%$ ). Nonpenumbral pattern was defined as the presence of larger core or small or absent penumbra. The study randomized patients into 4 groups based on perfusion results (penumbral versus nonpenumbral) and type of treatment (medical versus endovascular). Patients with confirmed LVO were randomly assigned within 8 hours after the onset of symptoms to undergo either mechanical embolectomy (Merci retriever or Penumbra system) or standard medical care.

3.2. Results. Embolectomy and standard medical care exhibited similar rates of mRS (3.9 versus 3.9, $P=0.99$ ). Moreover, there was no significant difference between embolectomy and standard medical care in patients with penumbral pattern versus nonpenumbral pattern. On the 3-month mRS, there was no interaction between the pretreatment imaging pattern and treatment assignment $(P=0.14)$. However, the final infarct volume was lower in patients with a favorable penumbral pattern regardless of treatment assignment.

3.3. Flaws. A number of limitations that question the trial conclusions should be considered [14-16]. First and foremost, MR RESCUE only included first-generation endovascular thrombectomy technologies, and higher efficacy retrievers were not utilized. Secondly, the small number of patients assigned to each of the 4 groups has likely underpowered this trial. Importantly, effective recanalization was not achieved in most endovascular group patients, regardless of penumbral imaging pattern. Only 16 of 64 patients (27\%) achieved TICI $2 \mathrm{~b}$ or 3 reperfusion. This rate is obviously not in line with acceptable treatment standards for acute ischemic stroke. The goal of any endovascular stroke intervention should be to achieve arterial recanalization. It is therefore impossible for MR RESCUE to comment on the efficacy of endovascular stroke therapy when only a minority of patients achieved arterial recanalization. 


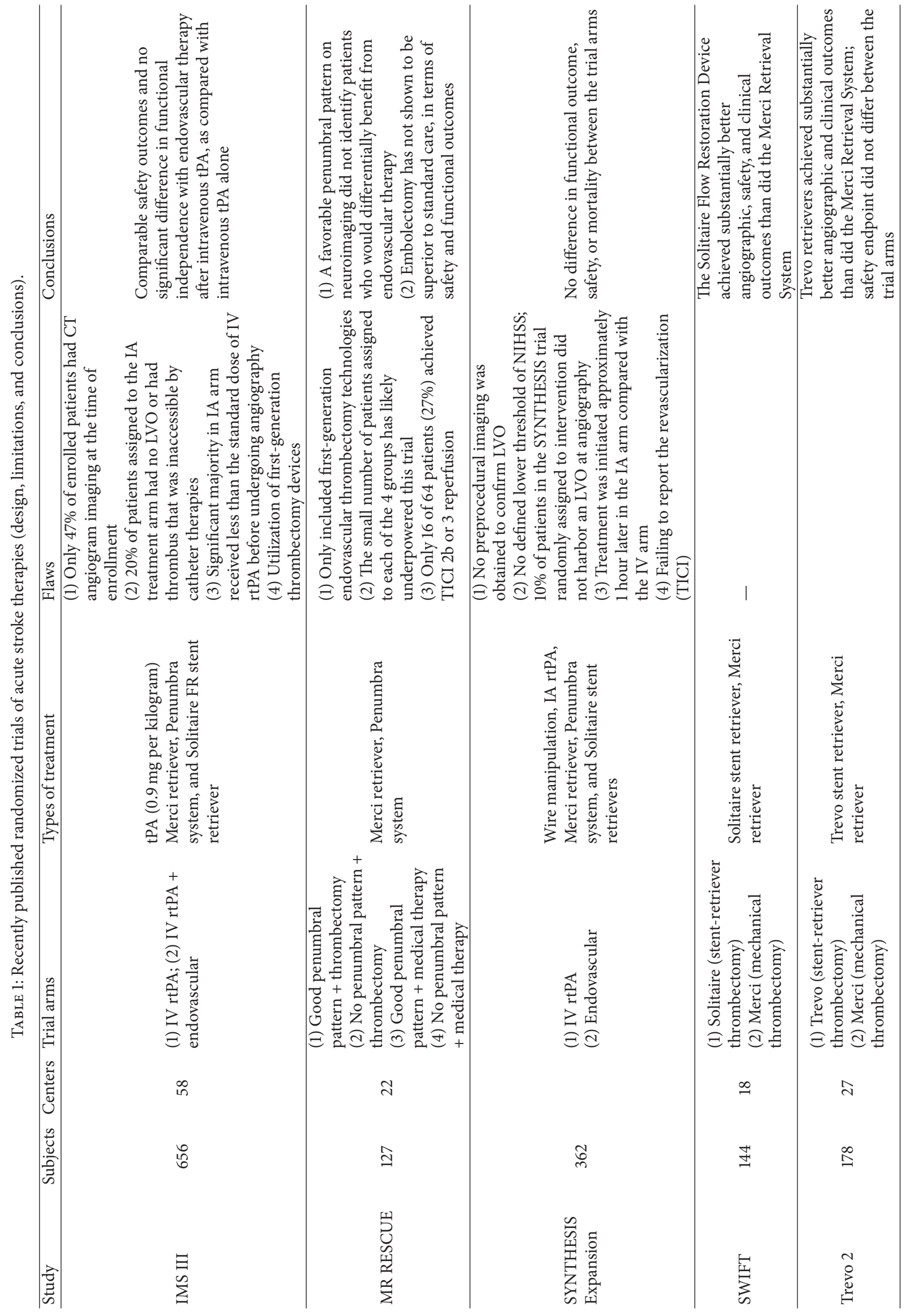




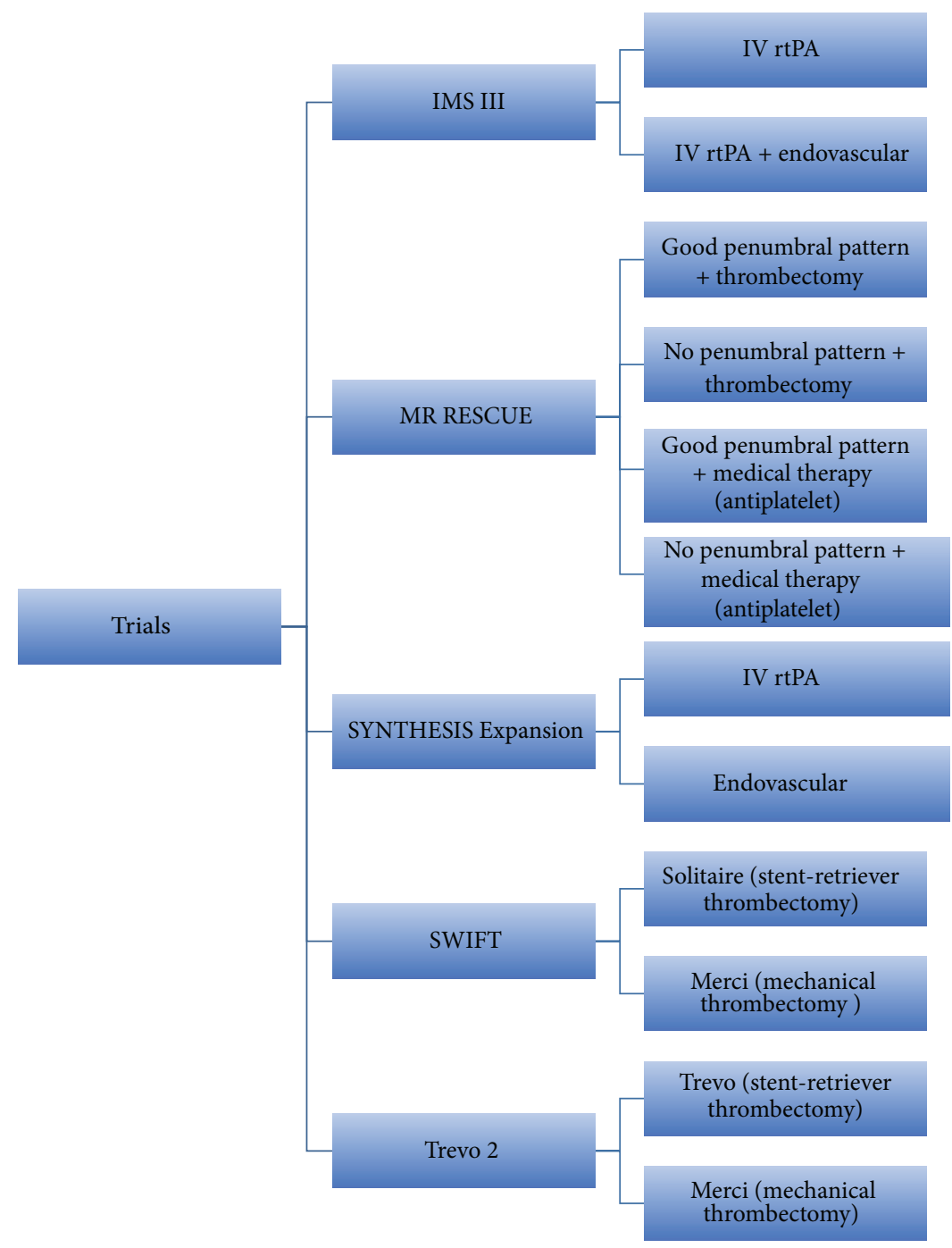

FIGURE 1: Trial arms of recently published randomized trials of acute stroke treatment.

3.4. Strengths. Unlike the IMS III or SYNTHESIS trials, pretreatment evaluation was more precise. CTA or MRA was used to depict LVO, and multimodal CT or MR imaging of the brain was used to evaluate penumbral status (Table 1 , Figure 1).

\section{SYNTHESIS Expansion}

4.1. Design. It is a multicenter Italian trial that randomly assigned 362 patients presenting with an AIS. The trial compared the treatment with IV rtPA within 4.5 hours of onset versus IA therapy within 6 hours of onset.

4.2. Results. There was no significant difference between both trial arms in terms of safety (rates of intracranial hemorrhage and death) or long-term outcomes.

4.3. Flaws. The trial had a number of weaknesses that question the validity of its results [14-16]. CTA or MRA was not obtained to confirm LVO or evaluate penumbra. Additionally, there was no lower threshold of NIHSS defined for study inclusion present. Around half of the patients enrolled had an NIHSS of 10 or less. Patients with NIHSS score as low as 2 were included; such patients are more likely to have a good recovery at 3 months regardless of the treatment given [16]. About $10 \%$ of patients assigned to intervention did not exhibit an LVO at angiography. Still, those patients were exposed to the potential risks of an intervention with injection of thrombolytics in the suspected target vessel.

Most of the patients in endovascular arm were treated with either wire manipulation or local thrombolysis. Only $10 \%$ received stent retrievers as a treatment. Additionally, the endovascular group received treatment 1 hour later than the IV tPA group, which might explain the comparable results of endovascular treatment and IV tPA treatment. Given this, it is expected that the SYNTHESIS study reconfirms the IMS III outcomes regarding the limited efficacy of 
endovascular treatment performed with outdated methods (Table 1, Figure 1).

\section{Recent Trials Showing Superiority of Stent Retrievers}

5.1. SWIFT. SWIFT was a multicenter, randomized, prospective, parallel-group trial with blinded primary endpoint ascertainment [17]. SWIFT was designed to provide definitive information on the efficacy and safety of the Solitaire Flow Restoration device in comparison to first-generation Merci retriever. It was the first trial to conduct a direct, randomized comparison of one mechanical thrombectomy device to another.

Patients were eligible if they had AIS with moderate to severe neurological deficits, harbored angiographically confirmed occlusions of proximal cerebral arteries. Inclusion criteria were NIHSS score $(\geq 8$ and $\leq 30)$ and ineligibility for or failure to respond to intravenous rtPA.

The primary efficacy outcome was achieved more often in the Solitaire group than it was in the Merci group (61\% versus 24). More patients had better 3-month neurological outcome with Solitaire than with Merci (58\% versus 33\%); 90-day mortality was lower in the Solitaire group than it was in the Merci group (17 versus 38). Thus, the Solitaire Flow Restoration Device was associated with substantially better angiographic, safety, and clinical outcomes compared to the Merci Retrieval System (Table 1, Figure 1).

5.2. Trevo 2. Trevo 2 was a randomized, prospective, controlled, multicenter, open-label trial [18]. The trial aimed to compare the efficacy and safety of mechanical thrombectomy using Trevo retriever with that of the Merci retriever in AIS. Patients were recruited from 26 sites in the USA and one in Spain. Participants had angiographically confirmed LVO strokes and NIHSS scores of 8-29 within $8 \mathrm{~h}$ of symptom onset. Eighty-six percent of patients in the Trevo group and $60 \%$ in the Merci group have shown successful recanalization, defined as TICI 2 or greater flow in the territory of the occlusion. The Trevo retrievers showed superior neurologic outcome compared to Merci device; however, the mortality rates were comparable.

The results of these 2 trials highlight again the superiority of stent retrievers to older devices in acute stroke intervention. Thus, any trial assessing the safety and efficacy of endovascular therapy should have stent retrievers as the main treatment modality (Table 1, Figure 1).

5.3. Our Experience. Two different studies on endovascular intervention for different age groups were conducted at the senior author institution $[19,20]$.

5.3.1. Young Patients with Large Vessel Occlusions. Young patients ( $<55$ years old) undergoing endovascular intervention for acute ischemic stroke were included in this study. Patients included had confirmed LVO, NIHSS greater than or equal to 5-8, and evidence of a large penumbra on CT perfusion. The mean admission NIHSS score was 14.1 (median 13.5). Intravenous rtPA was administered before initiation of endovascular therapy in half of the patients.

About one-third of the patients received secondgeneration retrievers, and the other two-thirds were treated with first-generation devices. Successful recanalization was achieved in as many as $93 \%$ of patients, and more than $90 \%$ achieved $\geq$ II TIMI. Favorable outcome mRS (0-2) was achieved in up to $73 \%$ of patients. Interestingly, secondgeneration retrievers (Solitaire) showed higher rates of favorable outcome compared to the first-generation devices. More importantly, endovascular treatment tended to be even more effective in younger patients $<35$ compared to those $>35$. These findings suggest that endovascular treatment for AIS has a particularly favorable outcome in young patients and support aggressive interventional strategies in this age group.

5.3.2. Septuagenarians and Above. A retrospective chart review was conducted to include patients over 75 years old treated for AIS. Inclusion criteria for intervention were a minimum NIHSS of 8, CT perfusion with ischemic penumbra on mean transient time and cerebral blood flow but preservation of cerebral blood volume, and detectable arterial occlusion on CT angiography.

Eight (16\%) patients underwent stent placement after intra-arterial thrombolysis, 10 (20\%) underwent balloon angioplasty, and seven (14\%) underwent both angioplasty and stent placement. Twenty-one (41\%) required only intraarterial thrombolytics. An improvement in thrombolysis score was noted in $67 \%$ of the patients. The average mRS score on discharge was 3.9. Symptomatic intracranial hemorrhage occurred in $6 \%$ of the patients. Two fatalities resulted from intraoperative vessel rupture (3.9\%).

Due to strict inclusion criteria for intervention given the increased risks of endovascular treatment in this age group, the patient population is rather small. Moreover, patient outcome at discharge may also not reflect ultimate outcome. Another limitation to our study is the lack of shift analysis of mRS, that is, assessment of mRS improvement rather than recording the good outcome only. Larger prospective randomized studies are required to investigate acute stroke interventions in this patient population.

Authors concluded that multimodal endovascular recanalization of AIS is a relatively safe treatment option in patients older than 75 years of age. However, careful patient selection by clinical and radiographic inclusion criteria is necessary for the successful management of stroke in this age group.

5.3.3. Evaluation of the Triage Protocol for Acute Ischemic Stroke. We conducted a retrospective review on 132 patients, 94 of which were undergoing CTP-guided and 38 undergoing time-guided (maximum $8 \mathrm{~h}$ from symptom onset) mechanical recanalization [21]. The study aimed at comparing the safety and efficacy of CTP-guided to time-guided mechanical recanalization in AIS. We observed no difference in the partial-to-complete recanalization rate between the CTP and the non-CTP group. However, the non-CTP group experienced a significantly higher rate of ICH. Multivariate 
analysis revealed CTP-guided patient selection to be an independent negative predictor of in-hospital mortality (OR $=3.2, P=0.01$ ), suggesting a potential benefit to using CTP for triage. Perfusion studies have now become the preferred triage method at our institution. We believe that, for stroke interventions to be effective, advanced imaging techniques such a CTP should be used to identify patients who can benefit from treatment.

\section{Conclusions}

IMS III, MR RESCUE, and SYNTHESIS Expansion trial results in the New England Journal of Medicine raised the doubts about the clinical value of intra-arterial (IA) therapy for the treatment of AIS. However, each has significant limitations that prevent its generalization to contemporary AIS treatment. Failure to adequately identify LVOs in IMS III and SYNTHESIS trials is a major drawback. Both IMS III and SYNTHESIS trials failed to use appropriate imaging technique (e.g., CTA, MRA) to confirm LVO. Moreover, salvageable brain evaluation with CT perfusion or MR was not conducted.

The second major drawback is the predominant use of the first-generation thrombectomy devices in the three trials. Subsequently, the revascularization rate did not meet the standards, particularly in MR RESCUE trial where it was dramatically worse than recent SWIFT and Trevo trials using second-generation devices.

Moreover, delays in treatment further biased the trials in IA therapy; treatment was initiated approximately 1-2 hours later in the IA arm compared with the IV arm.

Analysis of the IMS III, SYNTHESIS, and MR RESCUE studies was informative in terms of improvements that should be implemented in future studies. The future evaluation of endovascular therapy in AIS has to consider proper selection of patients (confirmed large vessel occlusion, evaluation of salvageable brain) and the homogenous use of up-todate treatment methods (e.g., second-generation retrievers). It is important to reiterate that when only patients with confirmed LVOs were included in the analysis of the IMS III, endovascular therapy showed a significant clinical benefit.

Our experience at our institution has confirmed remarkably high rates of arterial recanalization and favorable outcomes in young patients, presenting with AIS and LVO. Thus, aggressive intervention strategy in these patients is recommended. Furthermore, in patients older than 75 years, multimodal endovascular recanalization of AIS has proved to be a relatively safe treatment option. However, careful patient selection by clinical and radiographic inclusion criteria is necessary for the successful management of stroke in this age group.

\section{Conflict of Interests}

The authors declare that there is no conflict of interests regarding the publication of this paper.

\section{References}

[1] D. Lloyd-Jones, R. Adams, M. Carnethon et al., "Heart disease and stroke statistics-2009 update: a report from the American Heart Association Statistics Committee and Stroke Statistics Subcommittee," Circulation, vol. 119, no. 3, pp. 480-486, 2009.

[2] J. L. Saver, R. Jahan, E. I. Levy et al., "SOLITAIRE with the intention for thrombectomy (SWIFT) trial: design of a randomized, controlled, multicenter study comparing the SOLITAIRE Flow Restoration device and the MERCI Retriever in acute ischaemic stroke," International Journal of Stroke, vol. 9, no. 5, pp. 658-668, 2014.

[3] W. S. Smith, G. Sung, S. Starkman et al., "Safety and efficacy of mechanical embolectomy in acute ischemic stroke: results of the MERCI trial," Stroke, vol. 36, no. 7, pp. 1432-1438, 2005.

[4] J. P. Broderick, Y. Y. Palesch, A. M. Demchuk et al., "Endovascular therapy after intravenous t-PA versus t-PA alone for stroke," The New England Journal of Medicine, vol. 368, no. 10, pp. 893903, 2013.

[5] C. S. Kidwell, R. Jahan, J. Gornbein et al., "A trial of imaging selection and endovascular treatment for ischemic stroke," The New England Journal of Medicine, vol. 368, no. 10, pp. 914-923, 2013.

[6] A. Ciccone, L. Valvassori, and M. Nichelatti, "SYNTHESIS expansion: design of a nonprofit, pragmatic, randomized, controlled trial on the best fast-track endovascular treatment vs. standard intravenous alteplase for acute ischemic stroke," International Journal of Stroke, vol. 6, no. 3, pp. 259-265, 2011.

[7] S. Abilleira, P. Cardona, M. Ribo et al., "Outcomes of a contemporary cohort of 536 consecutive patients with acute ischemic stroke treated with endovascular therapy," Stroke: A Journal of Cerebral Circulation, vol. 45, no. 4, pp. 1046-1052, 2014.

[8] J. J. McCabe, T. J. Phillips, C. Phatouros et al., "Mechanical thrombectomy with the Solitaire $\mathrm{AB}$ device in large intracerebral artery occlusions," Journal of Medical Imaging and Radiation Oncology, vol. 57, no. 2, pp. 149-155, 2013.

[9] A. Ciccone, L. Valvassori, M. Nichelatti et al., "Endovascular treatment for acute ischemic stroke," The New England Journal of Medicine, vol. 368, no. 10, pp. 904-913, 2013.

[10] E. Broussalis, E. Trinka, W. Hitzl, A. Wallner, V. Chroust, and M. Killer-Oberpfalzer, "Comparison of stent-retriever devices versus the merci retriever for endovascular treatment of acute stroke," The American Journal of Neuroradiology, vol. 34, no. 2, pp. 366-372, 2013.

[11] A. Alshekhlee, D. J. Pandya, J. English et al., "Merci mechanical thrombectomy retriever for acute ischemic stroke therapy: literature review," Neurology, vol. 79, no. 13, supplement 1, pp. S126-S134, 2012.

[12] V. M. Pereira, J. Gralla, A. Davalos et al., "Prospective, multicenter, single-arm study of mechanical thrombectomy using solitaire flow restoration in acute ischemic stroke," Stroke, vol. 44, no. 10, pp. 2802-2807, 2013.

[13] P. Machi, V. Costalat, K. Lobotesis et al., "Solitaire FR thrombectomy system: Immediate results in 56 consecutive acute ischemic stroke patients," Journal of NeuroInterventional Surgery, vol. 4, no. 1, pp. 62-66, 2012.

[14] A. A. Khalessi, K. M. Fargen, S. Lavine, and J. Mocco, "Commentary: societal statement on recent acute stroke intervention trials: results and implications," Neurosurgery, vol. 73, no. 2, pp. E375-E379, 2013. 
[15] M. Mokin, A. A. Khalessi, J. Mocco et al., "Endovascular treatment of acute ischemic stroke: the end or just the beginning?" Neurosurgical Focus, vol. 36, no. 1, article E5, 2014.

[16] L. Pierot, J. Gralla, C. Cognard, and P. White, "Mechanical thrombectomy after IMS III, synthesis, and MR-RESCUE," The American Journal of Neuroradiology, vol. 34, no. 9, pp. 1671-1673, 2013.

[17] J. L. Saver, R. Jahan, E. I. Levy et al., "Solitaire flow restoration device versus the Merci Retriever in patients with acute ischaemic stroke (SWIFT): a randomised, parallel-group, noninferiority trial," The Lancet, vol. 380, no. 9849, pp. 1241-1249, 2012.

[18] R. G. Nogueira, H. L. Lutsep, R. Gupta et al., “Trevo versus Merci retrievers for thrombectomy revascularisation of large vessel occlusions in acute ischaemic stroke (TREVO 2): a randomised trial," The Lancet, vol. 380, no. 9849, pp. 1231-1240, 2012.

[19] N. Chalouhi, S. Tjoumakaris, R. M. Starke et al., "Endovascular stroke intervention in young patients with large vessel occlusions," Neurosurgical Focus, vol. 36, no. 1, article E6, 2014.

[20] G. M. Ghobrial, N. Chalouhi, L. Rivers et al., "Multimodal endovascular management of acute ischemic stroke in patients over 75 years old is safe and effective," Journal of NeuroInterventional Surgery, vol. 5, no. 1, pp. i33-i37, 2013.

[21] N. Chalouhi, G. Ghobrial, S. Tjoumakaris et al., "CT perfusionguided versus time-guided mechanical recanalization in acute ischemic stroke patients," Clinical Neurology and Neurosurgery, vol. 115, no. 12, pp. 2471-2475, 2013. 


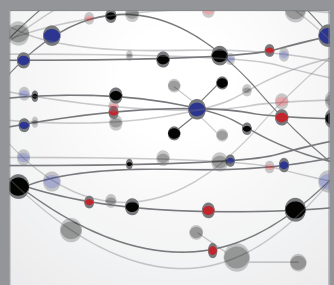

The Scientific World Journal
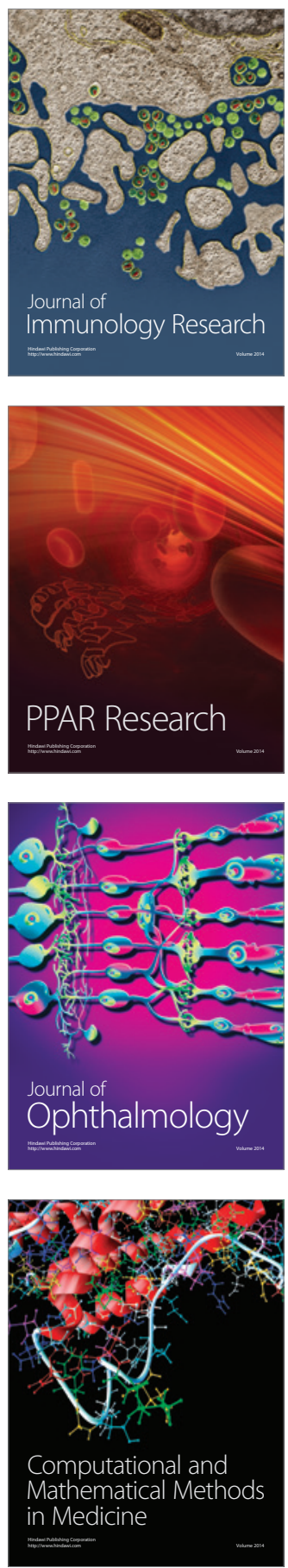

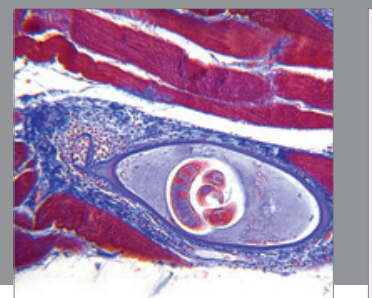

Gastroenterology

Research and Practice
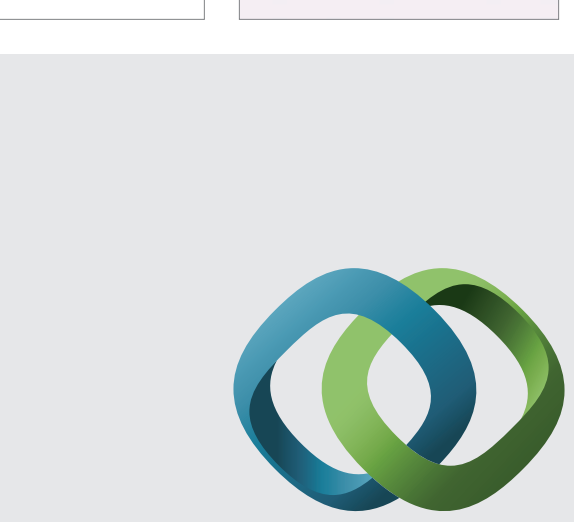

\section{Hindawi}

Submit your manuscripts at

http://www.hindawi.com
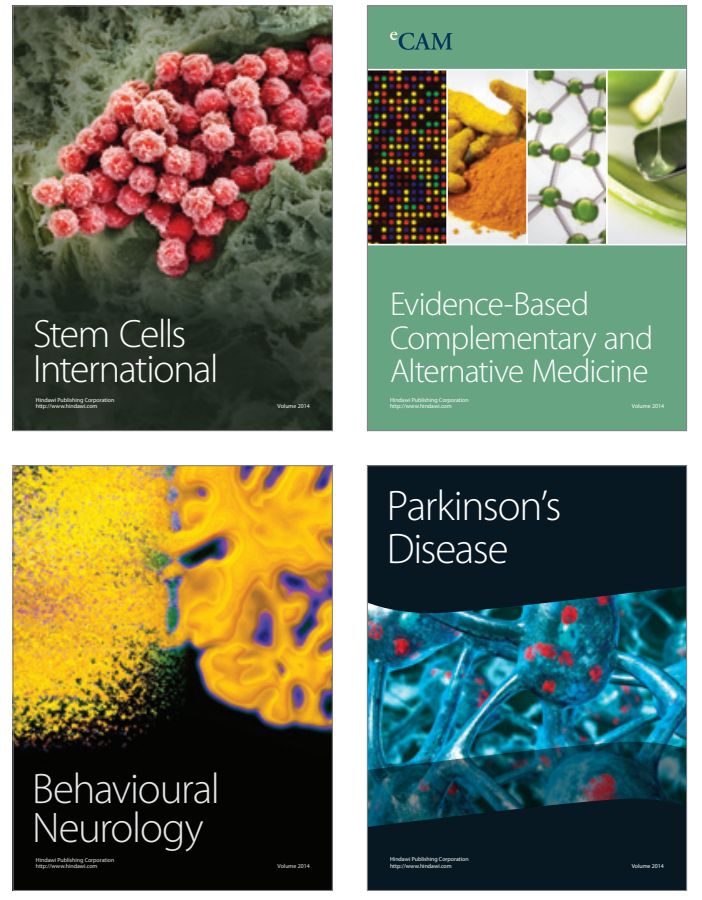
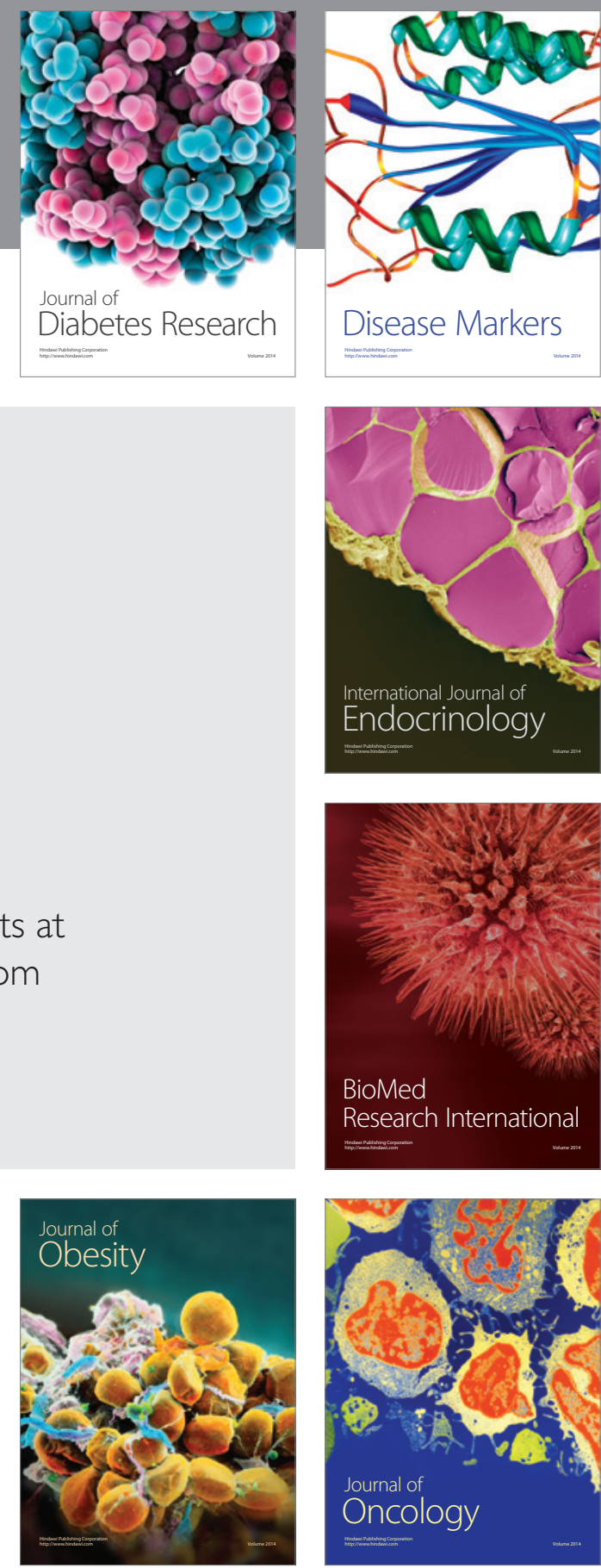

Disease Markers
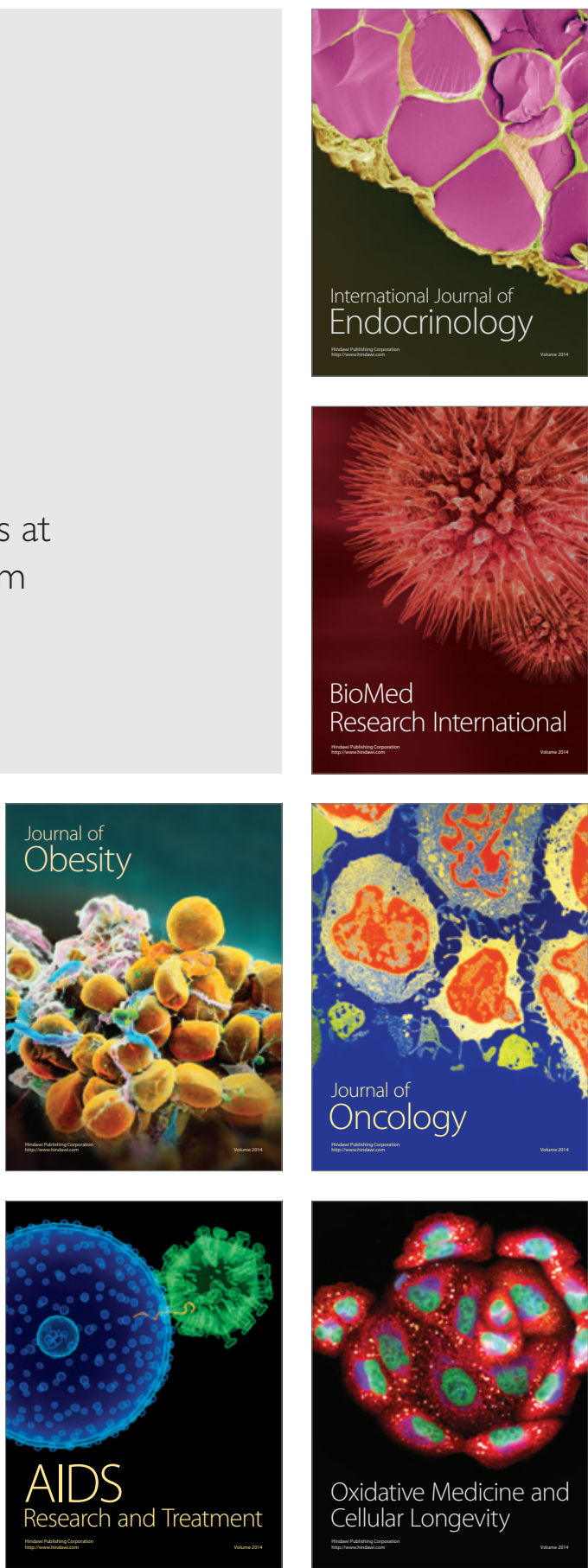